\title{
Inhibiting Vascular Endothelial Growth Factor in Injured Intervertebral Discs Attenuates Pain-Related Neuropeptide Expression in Dorsal Root Ganglia in Rats
}

\author{
Jun Sato ${ }^{1,2}$, Kazuhide Inage ${ }^{1}$, Masayuki Miyagi ${ }^{3}$, Yoshihiro Sakuma ${ }^{4}$, Kazuyo Yamauchi $^{1}$, Masao Koda ${ }^{1}$, \\ Takeo Furuya ${ }^{1}$, Junichi Nakamura ${ }^{1}$, Miyako Suzuki ${ }^{1}$, Go Kubota ${ }^{5}$, Yasuhiro Oikawa ${ }^{6}$, Takeshi Sainoh ${ }^{7}$, \\ Kazuki Fujimoto ${ }^{1}$, Yasuhiro Shiga ${ }^{1}$, Koki Abe ${ }^{1}$, Hirohito Kanamoto ${ }^{1}$, Masahiro Inoue ${ }^{1}$, Hideyuki Kinoshita ${ }^{1}$, \\ Masaki Norimoto ${ }^{1}$, Tomotaka Umimura ${ }^{1}$, Kazuhisa Takahashi ${ }^{1}$, Seiji Ohtori ${ }^{1}$, Sumihisa Orita ${ }^{1}$ \\ ${ }^{1}$ Department of Orthopedic Surgery, Chiba University Graduate School of Medicine, Chiba, Japan \\ ${ }^{2}$ Department of Orthopedic Surgery, Chiba Aoba Municipal Hospital, Chiba, Japan \\ ${ }^{3}$ Department of Orthopedic Surgery, Kitasato University Hospital, Tokyo, Japan \\ ${ }^{4}$ Department of Orthopedic Surgery, National Hospital Organization Chiba Medical Center, Chiba, Japan \\ ${ }^{5}$ Department of Orthopedic Surgery, Eastern Chiba Medical Center, Chiba, Japan \\ ${ }^{6}$ Department of Orthopedic Surgery, Chiba Children's Hospital, Chiba, Japan \\ ${ }^{7}$ Department of Orthopedic Surgery, Sainou Hospital, Toyama, Japan
}

Study Design: An experimental animal study.

Purpose: To evaluate effects of anti-vascular endothelial growth factor (VEGF) on the content and distribution of the calcitonin generelated peptide (CGRP) in the dorsal ganglia in a rat model.

Overview of Literature: Increased expression of VEGF in degenerative disc disease increases the levels of inflammatory cytokines and nerve ingrowth into the damaged discs. In animal models, increased levels of VEGF can persist for up to 2 weeks after an injury. Methods: Through abdominal surgery, the dorsal root ganglia (DRG) innervating L5/L6 intervertebral disc were labeled (FluoroGold neurotracer) in 24, 8-week old Sprague Dawley rats. The rats were randomly allocated to three groups of eight rats each. The antiVEGF group underwent L5/6 intervertebral disc puncture using a 26-gauge needle, intradiscal injection of $33.3 \mu \mathrm{g}$ of the pegaptanib sodium, a VEGF165 aptamer. The control-puncture group underwent disc puncture and intradiscal injection of $10 \mu \mathrm{L}$ saline solution, and the sham-surgery group underwent labeling but no disc puncture. Two rats in each group were sacrificed on postoperative days 1,7, 14, and 28 after surgery. L1-L6 DRGs were harvested, sectioned, and immunostained to detect the content and distribution of CGRP. Results: Compared with the control, the percentage of CGRP-positive cells was lower in the anti-VEGF group $(p<0.05 ; 40.6 \%$ and $58.1 \%$ on postoperative day $1,44.3 \%$ and $55.4 \%$ on day 7 , and $42.4 \%$ and $59.3 \%$ on day 14$)$. The percentage was higher in the control group compared with that of the sham group $(p<0.05$; sham group, $34.1 \%, 40.7 \%$, and $33.7 \%$ on postoperative days 1,7 , and 14 , respectively). Conclusions: Decreasing CGRP-positive cells using anti-VEGF therapy provides fundamental evidence for a possible therapeutic role of anti-VEGF in patients with discogenic lower back pain.

Keywords: Intervertebral discs; Back pain; Vascular endothelial growth factor

\footnotetext{
Received Nov 4, 2016; Revised Dec 26, 2016; Accepted Jan 26, 2017

Corresponding author: Kazuhide Inage

Department of Orthopedic Surgery, Graduate School of Medicine, Chiba University,

1-8-1 Inohana, Chuo-ku, Chiba 260-8670, Japan

Tel: +81-43-226-2117, Fax: +81-43-226-2116, E-mail: kazuhideinage@yahoo.co.jp
} 


\section{Introduction}

Nonspecific lower back pain (LBP) accounts for up to $85 \%$ of patients with chronic LBP. However, confirming a diagnosis and implementing an effective treatment for nonspecific LBP is difficult $[1,2]$. Therefore, clarifying its causes would be critical for advancing orthopedic practice, improving diagnosis, and identifying effective treatments. Fundamental research on the pathological pathways of nonspecific LBP has been actively pursued in recent years, with emerging evidence for a role of the intervertebral disc (IVD), intervertebral joints, spinal cord, muscle, and fascia in the development and maintenance of nonspecific LBP [3].

Of these possible sources of pain, the specific contribution of the IVD has been extensively considered and investigated in recent years [4]. In their study on discogenic LBP, Burke et al. [5] reported that the levels of inflammatory cytokines such as tumor necrosis factor (TNF)- $\alpha$, interleukin (IL)-6, and nerve growth factors (NGFs), and inflammatory mediators are increased in the intervertebral disc of patients with chronic discogenic LBP. Furthermore, Shinohara [6] reported abnormal elongation of free nerve endings within the IVD showing evidence of degeneration. On the basis of these results, a number of studies have evaluated the effectiveness of various treatments targeting the IVD for managing discogenic pain. In clinical research, treatment of discogenic LBP with TNF- $a$ inhibitors locally applied to the IVD is effective for controlling pain for up to 8 weeks after administration [7]. Of these novel therapies targeting inflammatory cytokines and NGF at the level of the IVD, studies investigating the role of vascular endothelial growth factor (VEGF) are of specific importance in our view.

VEGF is a vascular survival factor that promotes angiogenesis and vascular permeability [8]. The expression of VEGF is increased in degenerative IVDs [9] and can induce disc inflammation $[10,11]$ and facilitate nerve elongation [12]. Therefore, it is plausible that increased expression of VEGF in degenerative IVD disease can stimulate inflammation, contributing to the development and prolongation of pain. Treatment targeting the suppression of VEGF would be an effective means in this case for reducing the symptomology of discogenic LBP. Based on this assumption, the aim of our present study was to investigate how the administration of pegaptanib, a VEGF aptamer that inhibits VEGF function, would influence the distribution of pain-related neuropeptide (the calcitonin gene-related peptide, CGRP) in the dorsal ganglia (DRG) in a rat model of IVD injury.

\section{Materials and Methods}

All protocols for the animal procedures were approved by our institution's ethics committees, and the study followed the National Institutes of Health Guidelines for the Care and Use of Laboratory Animals (1996 revision).

\section{Retrograde FluoroGold detection of DRG neurons}

Twenty-four male, 8-week-old, Sprague Dawley rats, weighing 250-300 g were included in the study. All animals were anesthetized by intraperitoneally administering sodium pentobarbital ( $40 \mathrm{mg} / \mathrm{kg})$, using aseptic technique, which was maintained throughout the experimental procedures. Under microscopic guidance, a ventral longitudinal incision was made at the midline of the abdomen of each rat to expose the L5/6 IVD.

The rats were randomly allocated to groups $(n=8$, each) as follows: sham-surgery, control-puncture, and anti-VEGF. Rats in the sham-surgery group underwent abdominal incision and exposure of the L5/L6 IVD. A midline abdominal incision was made with the rat supine. The retroperitoneum was incised along the left margin of the aorta, and the ventral surface of the L5-L6 intervertebral disc was exposed between the aorta and the left psoas muscle. Rats in the control-puncture group underwent 10 punctures of the IVD using a 26-gauge needle and intradiscal injection of $10 \mu \mathrm{L}$ of saline. The injection wound was immediately sealed to prevent leakage. Rats in the anti-VEGF group underwent this same procedure, except for the intradiscal injection of $33.3 \mu \mathrm{g}$ of pegaptanib (Pfizer Inc., Ann Arbor, MI, USA), which was mixed with saline (total volume, $10 \mu \mathrm{L}$ ); the injection wound was immediately sealed to prevent leakage.

The FluoroGold (FG) neurotracer (Fluorochrome, Denver, CO, USA) was applied to the surface of the L5/6 IVD of the 24 rats to label the neurons of the DRG innervating the discs. Following this application, the abdominal incision was closed.

Two animals in each group were sacrificed on postoperative days 1, 7, 14, and 28. Animals were anesthetized with intraperitoneal administration of sodium pentobarbital (40 mg/kg), which was perfused transcardially with 
$0.9 \%$ saline followed by $500 \mathrm{~mL}$ of $4 \%$ paraformaldehyde in phosphate buffer (0.1 M, pH 7.4). Levels L1 through L6 DRGs were bilaterally resected. For immunofluorescence analysis, specimens were immersed in a fixative solution (0.01 M phosphate-buffered saline [PBS] containing $20 \%$ sucrose) for 20 hours at $4^{\circ} \mathrm{C}$. Each ganglion was then sectioned into 10 - $\mu$ m-thick slices using a cryostat, and the sections were mounted on poly-1-lysine-coated slides.

Immunohistochemical analysis of CGRP expression required quenching of endogenous tissue peroxidase activity in a solution of $0.01 \mathrm{M}$ PBS containing $0.3 \%$ hydrogen peroxide for 30 minutes. Specimens were then treated for 90 minutes at room temperature in a blocking solution of $0.01 \mathrm{M}$ PBS, $0.3 \%$ Triton X-100, and 3\% skim milk. To stain the DRGs, sections were reacted with a rabbit antibody against CGRP (1:1000; Chemicon, Temecula, CA, USA) diluted in a blocking solution and were incubated for 20 hours at $4^{\circ} \mathrm{C}$. To detect CGRP in the DRGs, sections were incubated with a goat anti-rabbit Alexa 488 fluorescent antibody conjugate (1:400; Molecular Probes, Eugene, OR, USA). Sections were examined using a fluorescence microscope to count the numbers of FG-labeled neurons and CGRP-immunoreactive (-IR) neurons in 10 randomly selected areas of DRGs from each level. To standardize the procedures, the numbers of CGRP-IR and FG neurons per $0.45-\mathrm{mm}^{2}$ area were counted using a counting grid magnified $400 \times$. The cross-sectional areas of the CGRP-IR neurons in all animals were measured, and their distributions were compared.

\section{Statistical analysis}

At each level (L1-L6), the proportion (\%) of FG-labeled CGRP-IR neurons per total FG-labeled cells was calculated. Between-level and between-group comparisons were evaluated using nonrepeated analysis of variance, with Bonferroni post-hoc correction for multiple comparisons. The level of significance was defined a priori as $p<0.05$.

\section{Results}

At DRG levels, L1 through L6, FG-labeled DRG neurons innervating the L5/6 IVD and FG-labeled DRG neurons indicating routes of FG transport from the IVD were present bilaterally. In all DRG specimens, at all levels and in all three groups, the majority of cells was FG positive,

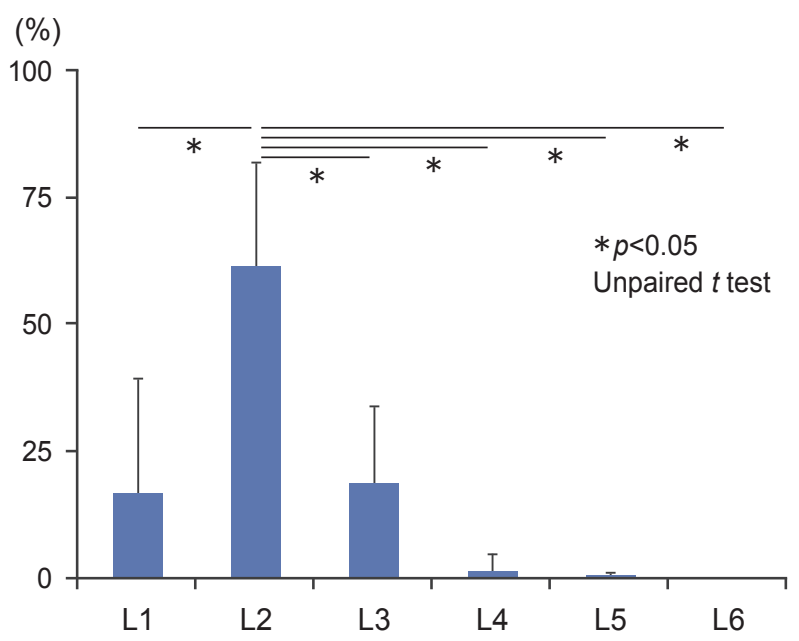

Fig. 1. The average numbers of FluoroGold (FG)-labeled DRG neurons from L1 through L6 levels in the three groups. DRG, dorsal root ganglia (expressed as the mean \pm standard error).

and the ratio of FG-positive cells to other cells was significantly higher at L2 (Fig. 1). A representative example of FG-labeled, CGRP-positive cells is shown in Fig. 2.

Table 1 and Fig. 3 show the percentage of FG-labeled CGRP-IR neurons per FG-labeled neurons in the L2 level. Compared with the sham-surgery group, the ratio of FG positive to CGRP-positive cells in the L2 DRG increased on postoperative days 1,7 , and 14 in both groups that underwent disc puncture $(p<0.05)$, with no between-group differences on postoperative day 28. Compared with the control-puncture group, the ratio of FG positive to CGRPpositive cells in the L2 DRG was significantly lower in the anti-VEGF group on postoperative days 1,7 , and 14 $(p<0.05)$, with no between-group differences on day 28 . All other measures were comparable between the controlpuncture and anti-VEGF groups.

\section{Discussion}

We show here that IVD puncture increased CGRP expression in the DRGs at levels L1-L6 through postoperative day 14. IVD puncture in rodents increases the number of CGRP-positive cells in the DRGs in the dominant region of damaged IVDs $[8,13,14]$. The findings of increased CGRP-positive cells indicate elevated levels of inflammatory cytokines such as IL-6, TNF- $\alpha$, or NGF in the IVD caused by a local inflammatory reaction to the puncture $[15,16]$. Sensitization of CGRP expression by these elevated levels of proinflammatory mediators was detected in the annulus fibrosus of the IVD [17]. These local sig- 

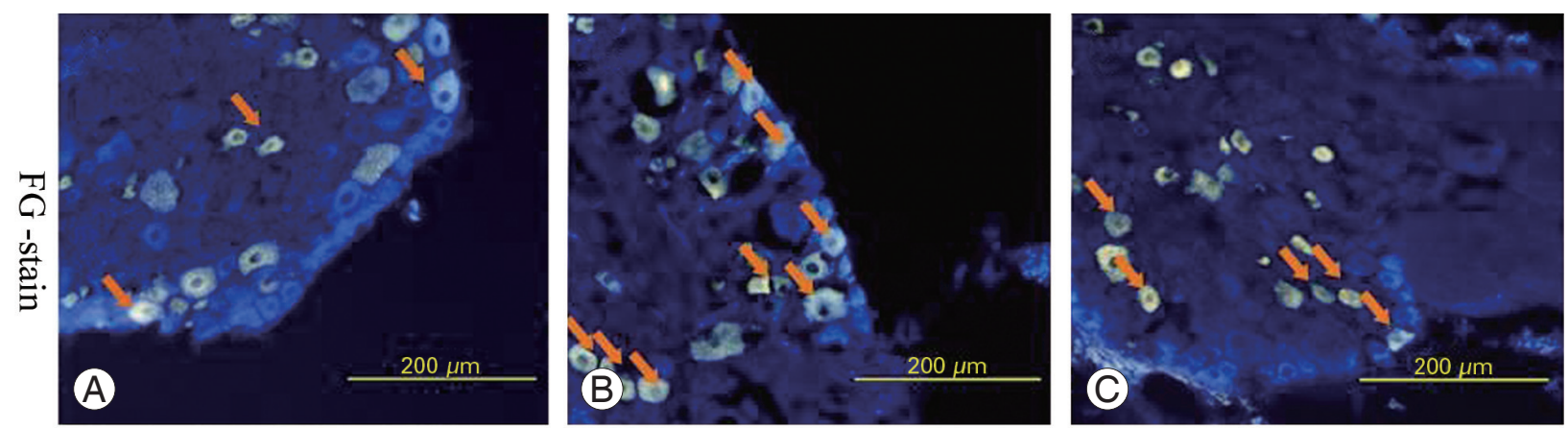

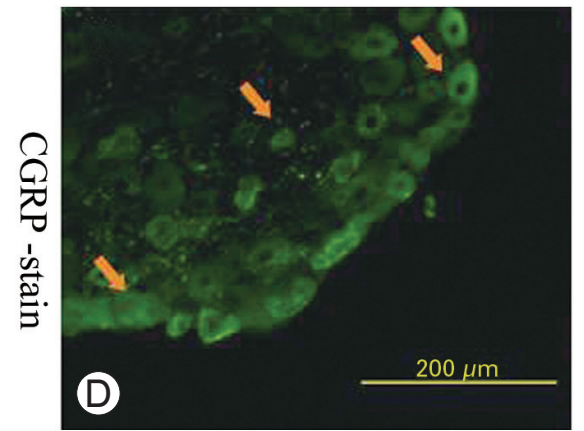

Non-puncture

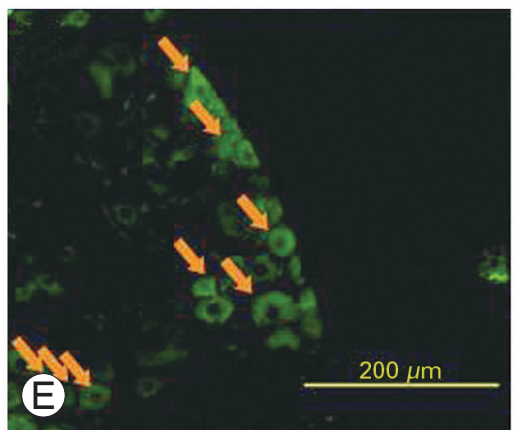

Puncture

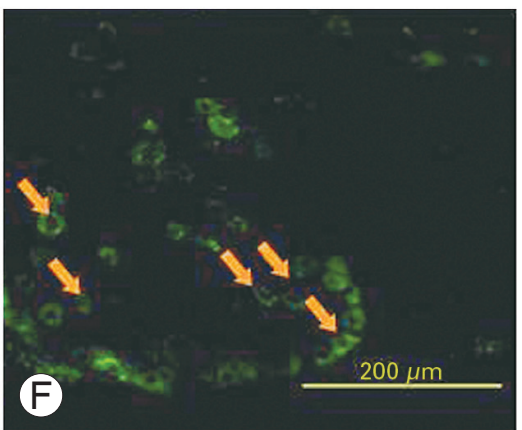

Anti-VEGF

\section{: CGRP-ir FG-labeled DRG neuron}

Fig. 2. FluoroGold (FG)-labeled (A) and calcitonin gene-related peptide (CGRP)-immunoreactive (-IR) (D) DRG in the sham-surgery group. FGlabeled (B) and CGRP-IR (E) DRG neurons in the control-puncture group. FG-labeled (C) and CGRP-IR (F) DRG neurons in the anti-VEGF group. Arrows indicate FG-labeled, CGRP-IR DRG neurons. Note that panels (A) and (D), (B) and (E), and (C) and (F) show the same section. DRG, dorsal root ganglia; VEGF, vascular endothelial growth factor.

Table1. The ratio of FG positive to CGRP-positive cells mean (standard deviation)

\begin{tabular}{lcccc} 
Mean (standard deviation) & 1 day & 7 day & 14 day & 28 day \\
Non-puncture & $34.1(5.3)$ & $40.7(4.8)$ & $33.3(13.4)$ & $41.1(17.7)$ \\
\hline Puncture & $58.1(11.3)$ & $55.3(5.5)$ & $59.3(8.5)$ & $47.0(6.9)$ \\
\hline Anti-VEGF & $40.6(7.0)$ & $44.3(8.3)$ & $42.4(6.2)$ & $44.5(9.8)$ \\
\hline
\end{tabular}

FG, FluoroGold; CGRP, the calcitonin gene-related peptide; VEGF, vascular endothelial growth factor.

nals can be transmitted via the primary afferent nerve to eventually increase the expression of CGRP throughout the dominant region of DRGs [14]. This mechanism can explain our finding of increased CGRP-positive cells in the DRGs within the dominant region of a damaged IVD.

Compared with the puncture group, the levels of CGRP-positive cells were significantly reduced in the antiVEGF group from postoperative days 1 through 14, indicating suppression of pain-related peptides in the sensory nerve roots by the administration of a VEGF inhibitor. This inhibition of VEGF may be important in modifying the mechanisms of the development of discogenic pain. Specifically, disc puncture produces a local inflammatory reaction, inducing an increase in VEGF, which eventually causes reinflammation by acting on local VEGF receptors (VEGFR) $[9,13]$. Among the VEGF receptors, VEGFR-1 may be specifically associated with the "re-inflammation" cycle, which would prolong pain through the reactivation of macrophages after a refractory period $[9,10]$. The putative role of VEGFR-1 in prolonging pain is supported by findings demonstrating the effectiveness of targeted inhibition of VEGFR-1 to suppress arthritic symptoms in a mouse model of rheumatoid arthritis [18].

We assumed that pegaptanib would uniquely act on VEGFR-1 [14]. Therefore, we propose the following mechanism to explain our results. Intervertebral disc 
(\%)

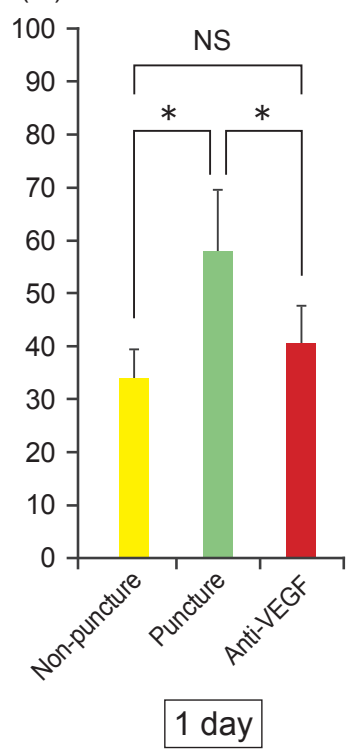

(\%)

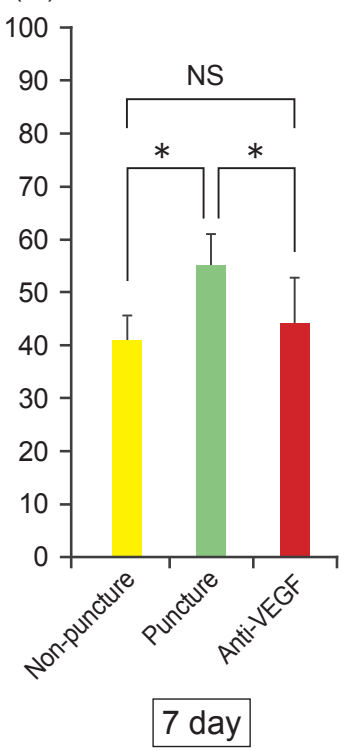

(\%)

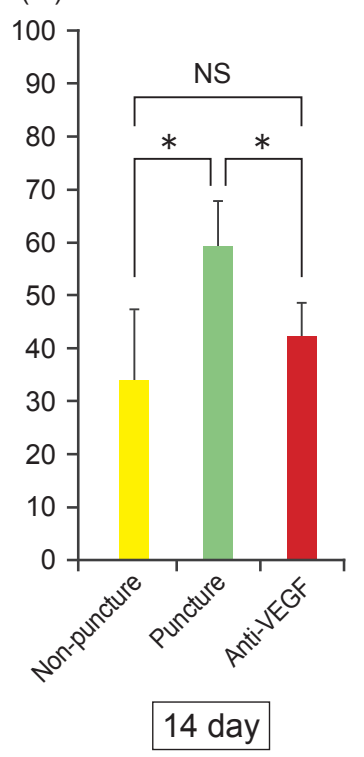

(\%)

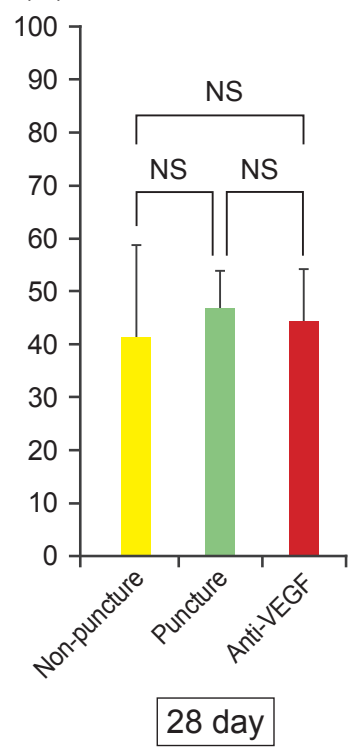

$* p<0.05 \quad$ Nonrepeated measures analysis of variance with Bonferroni post hoc correction.

Fig. 3. The ratio of FluoroGold (FG)-labeled and calcitonin gene-related peptide (CGRP)-positive neurons to the number of FG-labeled neurons at the L2 level, and the numbers of DRGs 1, 7, 14, and 28 days postpuncture. NS, not significant; VEGF, vascular endothelial growth factor; DRG, dorsal root ganglia.

puncture would cause a local inflammatory reaction, elevating VEGFR levels and triggering increased expression of CGFP. The administration of pegaptanib would uniquely act on VEGFR-1, suppressing the transition and activation of inflammatory cells such as VEGFR1mediated macrophages and, thereby, prevent reinflammation as well as the suppression of CGRP in the dominant sensory nerve. Pegaptanib therapy in clinical practice is safe and effective for the treatment of age-related macular degeneration. To our knowledge, there are no reports of severe side effects caused by the therapeutic applications of pegaptanib.

The limitations of our study must be considered when interpreting the present results. First, pegaptanib was administered as single dose, which should be considered in future research. Second, the injured IVD is a model of acute inflammation, which is insufficient for studying the pathology of chronic human LBP. Further research is required to evaluate its effectiveness and mechanisms in a model of disc degeneration. Third, the model did not consider continuous load application such as that experienced by humans when they stand. Therefore, the present model is inadequate for evaluating the chronic phase of pain. An appropriate model for degenerative IVD, which satisfies the conditions of prolonged loading during standing and cyclical loading while walking has yet to be developed and is the focus of our future research. Finally, we evaluated the effects of anti-VEGF on pain indirectly by quantifying the expression of CGRP in the DRGs. We are planning to include behavioral evaluations in future studies.

\section{Conclusions}

Local administration of a VEGF inhibitor after puncturing an IVD in a rat model effectively suppressed the expression of pain peptides for up to 14 days. We propose therefore that LBP, originating from injury to the IVD, may be ameliorated by suppressing VEGF activity. This provides a new therapy for managing disc-related LBP. Future research is required to determine the optimal dosage and to quantify the association between the suppression of VEGF behavioral responses to pain.

\section{Conflict of Interest}

No potential conflict of interest relevant to this article was reported. 


\section{References}

1. Koes BW, van Tulder MW, Thomas S. Diagnosis and treatment of low back pain. BMJ 2006;332:1430-4.

2. Lee S, Moon CS, Sul D, et al. Comparison of growth factor and cytokine expression in patients with degenerated disc disease and herniated nucleus pulposus. Clin Biochem 2009;42:1504-11.

3. Schwarzer AC, Aprill CN, Derby R, Fortin J, Kine G, Bogduk N. The relative contributions of the disc and zygapophyseal joint in chronic low back pain. Spine (Phila Pa 1976) 1994;19:801-6.

4. Ohtori S, Orita S, Yamauchi K, et al. Do physical symptoms predict the outcome of surgical fusion in patients with discogenic low back pain? Asian Spine J 2016;10:509-15.

5. Burke JG, Watson RW, McCormack D, Dowling FE, Walsh MG, Fitzpatrick JM. Intervertebral discs which cause low back pain secrete high levels of proinflammatory mediators. J Bone Joint Surg Br 2002;84:196201.

6. Shinohara H. Lumbar disc lesion, with special reference to the histological significance of nerve endings of the lumbar discs. Nihon Seikeigeka Gakkai Zasshi 1970;44:553-70.

7. Sainoh T, Orita S, Miyagi M, et al. Single intradiscal administration of the tumor necrosis factor-alpha inhibitor, etanercept, for patients with discogenic low back pain. Pain Med 2016;17:40-5.

8. Shibuya M. Differential roles of vascular endothelial growth factor receptor-1 and receptor- 2 in angiogenesis. J Biochem Mol Biol 2006;39:469-78.

9. Lu XY, Ding XH, Zhong LJ, Xia H, Chen XD, Huang $\mathrm{H}$. Expression and significance of VEGF and p53 in degenerate intervertebral disc tissue. Asian Pac J Trop Med 2013;6:79-81.

10. Kim JH, Studer RK, Vo NV, Sowa GA, Kang JD. p38 MAPK inhibition selectively mitigates inflamma- tory mediators and VEGF production in AF cells cocultured with activated macrophage-like THP-1 cells. Osteoarthritis Cartilage 2009;17:1662-9.

11. Kim JH, Studer RK, Sowa GA, Vo NV, Kang JD. Activated macrophage-like THP-1 cells modulate anulus fibrosus cell production of inflammatory mediators in response to cytokines. Spine (Phila Pa 1976) 2008; 33:2253-9.

12. Jin K, Zhu Y, Sun Y, Mao XO, Xie L, Greenberg DA. Vascular endothelial growth factor (VEGF) stimulates neurogenesis in vitro and in vivo. Proc Natl Acad Sci U S A 2002;99:11946-50.

13. Ramakrishnan S, Anand V, Roy S. Vascular endothelial growth factor signaling in hypoxia and inflammation. J Neuroimmune Pharmacol 2014;9:142-60.

14. Luttun A, Tjwa M, Moons L, et al. Revascularization of ischemic tissues by PlGF treatment, and inhibition of tumor angiogenesis, arthritis and atherosclerosis by anti-Flt1. Nat Med 2002;8:831-40.

15. Miyagi M, Ishikawa T, Orita $S$, et al. Disk injury in rats produces persistent increases in pain-related neuropeptides in dorsal root ganglia and spinal cord glia but only transient increases in inflammatory mediators: pathomechanism of chronic diskogenic low back pain. Spine (Phila Pa 1976) 2011;36:2260-6.

16. Ohtori S, Takahashi K, Chiba T, Yamagata M, Sameda $\mathrm{H}$, Moriya $\mathrm{H}$. Substance $\mathrm{P}$ and calcitonin generelated peptide immunoreactive sensory DRG neurons innervating the lumbar facet joints in rats. Auton Neurosci 2000;86:13-7.

17. Aoki Y, Ohtori S, Ino H, et al. Disc inflammation potentially promotes axonal regeneration of dorsal root ganglion neurons innervating lumbar intervertebral disc in rats. Spine (Phila Pa 1976) 2004;29:2621-6.

18. Ng EW, Adamis AP. Anti-VEGF aptamer (pegaptanib) therapy for ocular vascular diseases. Ann N Y Acad Sci 2006;1082:151-71. 